\title{
TOLERÂNCIA A ALUMÍNIO E EFICIÊNCIA A FÓSFORO EM MILHO E ARROZ: CARACTERÍSTICAS INDEPENDENTES $\left({ }^{1}\right)$
}

\author{
PEDRO ROBERTO FURLANI( ${ }^{2,3}$ ) E ANGELA MARIA CANGIANI FURLANI(2,3)
}

\begin{abstract}
RESUMO
Oito experimentos foram realizados em solução nutritiva, em casa de vegetaçăo localizada no Centro Experimental do Instituto Agronômico de Campinas, em 1982-86 , com o objetivo de avaliar cem linhagens de arroz e quarenta de milho quanto à eficiência e utilizaçăo de fósforo na ausência de A, e quanto à tolerância a A em baixo P. Verificou-se, através de coeficiente de correlação (r), se a tolerância a A se relacionava com a eficiência a $\mathrm{P}$, avaliadas por parâmetros confiáveis previamente estabelecidos. As linhagens de arroz e milho foram agrupadas em nove classes cujos limites foram determinados pelo intervalo de confiança da média do índice de eficiência a Pe da média do índice de tolerância a A. As linhagens de arroz de sequeiro mostraram-se superiores quanto à tolerância a $\mathbf{A}, \theta$ as de arroz irrigado, quanto à eficiência a P. Dez linhagens de arroz de sequeiro e cinco de arroz irrigado foram selecionadas como eficientes a $\mathrm{P}$ e tolerantes a Al. As linhagens de milho tenderam a uma inversão nos índices de avaliação das duas características, sendo três selecionadas como altamente tolerantes a $\mathrm{Al}$, mas intermediárias quanto à eficiência a $\mathrm{P}$. Para o material genético de arroz e milho, os coeficientes de correlação (r) entre os respectivos índices indicaram que as referidas características são independentes nas plantas.
\end{abstract}

Termos de indexação: arroz, milho, eficiência a P, tolerância a A.

\section{ABSTRACT \\ ALUMINUM TOLERANCE AND PHOSPHORUS EFFICIENCY IN MAIZE AND RICE: INDEPENDENT TRAITS}

Eight experiments were carried out in nutrient solutions under greenhouse conditions at the Instituto Agronômico Experimental Center, located at Campinas, State of São Paulo, Brazil. The main objective of this study was to determine the relationship between P-use efficiency and Al-tolerance of 50 lowland rice cultivars, 50 upland rice cultivars, and $\mathbf{4 0}$ maize inbred lines. Using the $99.9 \%$ confidence intervaT for the mean values of P-efficiency and Al-tolerance, respectively for each crop group, the genotypes were classified in nine classes: I. Efficient to $P$ and tolerant to A (ET);

(1) Trabalho financiado pelos Convênios EMBRAPASAA e FINEP/FDCT/SAA. Apresentado no XXII Congresso Brasileiro de Ciência do Solo, 23 a 31 de julho de 1989, Recife (PE). Recebido para publicação em 20 de novembro de 1990 e aceito em 14 de agosto de 1991.

(2) Seção de Fertilidade do Solo e Nutrição de Plantas, Instituto Agronômico de Campinas (IAC). Caixa Postal 28, 13001 Campinas (SP).

(3) Com bolsa de pesquisa do CNPq. 
II. Efficient to P and intermediate to A (El); III. Efficient to P and non-tolerant to A (ENT); IV. Intermediate to $P$ and tolerant to $A$ (IT); $\mathbf{V}$. Intermediate to $P$ and intermediate to A (II); VI. Intermediate to P and non-tolerant to A (INT); VI. Non-efficient to P and tolerant to A (NET); VIII. Non-efficient to P and intermediate to $A$ (NEI); and, $(X$. Non-efficient to $P$ and non-tolerant to $A$ (NENT). The non-efficient or non-tolerant, the intermediate, and the efficient or tolerant are those classes whose values are lesser, equal or greater than the confidence interval, respectively. In rice, the upland cultivars were more tolerant to $A$ but less efficient to $P$ than the lowland cultivars. Ten upland and five lowland rice cultivars could be classified as efficient to $P$ and tolerant to $A$ (ET). In maize, an inverse relationship was found between efficiency to $P$ and tolerance to A. Three inbred lines could be classified as intermediate to $P$ and tolerant to A (IT). For the genetic material used in this study, it was found a poor correlation between the two plant traits indicating that they are independent.

Index terms: rice, corn, P-efficiency; A-tolerance.

\section{INTRODUÇÃO}

Existe uma idéia generalizada de que a tolerância a Al e a eficiência na absorção de $P$ são características genéticas inseparáveis na planta.

Entretanto, ocorre que uma planta tolerante a Al consegue desenvolver seu sistema radicular em profundidade, apesar do ambiente hostil, e explorar maior volume de solo em busca de água e nutrientes. Uma planta tolerante a Al apresenta maior conteúdo não só de $\mathrm{P}$ como de todos os demais nutrientes.

Os mecanismos fisiológicos e bioquímicos envolvidos na tolerância a aluminio variam entre espécies e cultivares, e não estão ainda bem estabelecidos.

Várias hipóteses têm sido propostas com base em resultados experimentais, para explicar as diferenças nas plantas quanto a essa característica, como a indução à variação do pH da rizosfera; o mecanismo de absorção e translocação de Al; a complexação do Al em compostos orgânicos; as interações com os nutrientes; a capacidade de troca de cátions das raízes (FOY et al., 1978; RHUE, 1979).

A hipótese da elevação do $\mathrm{pH}$ da rizosfera foi levantada em conseqüência de resultados experimentais em tomate (FOY et al., 1973); arroz (HOWELER \& CADAVID, 1976), trigo, cevada e triticale (MUGWIRA et al., 1976), evidenciando um mecanismo de tolerância que bloquearia a absorção e transporte de Al ou proveria a precipitação de Al na rizosfera. Neste caso, as plantas tolerantes revelavam menores concentrações de Al na parte aérea. Plantas tolerantes que apresentam concentrações elevadas de Al nos tecidos da parte aérea e, mesmo assim, conseguem manter niveis adequados de $P$, parecem possuir um sistema tampão de ácidos inorgânicos predominantemente (JONES, 1961; CLARKSON, 1966; RHUE, 1979).

Interação do $\mathrm{Al}$ com os nutrientes $\mathrm{P}, \mathrm{Ca}, \mathrm{K}$ e $\mathrm{Mg}$ também podem estar envolvidos nos mecanismos de tolerância e variam de importância conforme a espécie ou cultivar. Estudando os mecanismos da toxicidade de Al e o metabolismo de $P$, FOY et al. (1978) conclúram que, em trigo e cevada, diferenças na tolerância ao Al poderiam ser só eventualmente explicadas por diferentes interações Al-P na 
planta. Outros pesquisadores verificaram que em arroz (HOWELER \& CADAVID, 1976), trigo, cevada e triticale (MUGWIRA et al., 1980), os teores de $\mathrm{P}, \mathrm{Ca}, \mathrm{Mg}$ e K eram mais altos nas plantas tolerantes, podendo as'diferenças na distribuição desses nutrientes contribuir na seleção dos cultivares. CASTELLS et al. (1985) utilizaram a taxa de absorção de fósforo como critério de seleção de genótipos de soja tolerantes a alumínio.

Neste trabalho são relatados e correlacionados dados de estudos em casa de vegetação, para seleção de grande número de linhagens de arroz e milho quanto à tolerância a Al e eficiência a $\mathrm{P}$, sendo esses estudos de seleção feitos independentemente um do outro. $O$ objetivo foi verificar, através de coeficientes de correlação, se a tolerância a Al se relacionava com a eficiência a P, para cem linhagens de arroz de sequeiro irrigado e quarenta de milho.

\section{MATERIAL E MÉTODOS}

Oito experimentos foram realizados em solução nutritiva, em casa de vegetação do Centro Experimental do Instituto Agronômico de Campinas, no período 1982-86: três deles com arroz de sequeiro e arroz irrigado para estudo de cem genótipos quanto à eficiência na absorção e utilização de fósforo, na ausência de Al. O método adotado está descrito em FURLANI et al. (1983) e FURLANI \& FURLANI (1988), referente à identificação dos genótipos utilizados, ao sistema de cultivo, à composição de soluções nutritivas e ao nivel adequado de $P$ para seleção de plantas de arroz. Outros dois experimentos foram realizados, com quarenta linhagens de milho, com os mesmos objetivos, estando os detalhes do método em FURLANI et al. (1985) e FURLANI \& FURLANI (1988). Finalmente, os outros três experimentos foram efetuados para seleção dos mesmos genótipos de arroz e milho quanto à tolerância a Al. O método definido, bem como o sistema de cultivo, estão relatados nos trabalhos de FURLANI \& HANNA (1984), FURLANI et al. (1986) e FURLANI \& FURLANI (1988).

A eficiência na absorção de $P$ foi avaliada através das variáveis: matéria seca (MS), conteúdo de fósforo, relação de eficiência (RE) e índice de eficiência de utilização (IE), sendo RE = MS total/P total, e IE $=M S \times R E$. O índice IE foi proposto por SIDDIQI \& GLASS (1981).

A tolerância a alumínio foi estimada pelo índice de tolerância relativa (ITR), calculado diferentemente para as duas espécies, através das expressões:

\section{Arroz}

onde:

$$
\text { ITR }=\left[\left(C^{2} R_{x}-C R R_{s}\right) /\left(C R R_{t}-C R R_{s}\right)\right] \times 4+1
$$

CRR é o comprimento relativo da radícula e representa a relação entre os valores do comprimento da radícula (CR) de plantas crescidas na presença de $20 \mathrm{mg} /$ litro de alumínio $(C R+A)$ e na ausência de alumínio (CR-A) respectivamente.

$$
\mathrm{CRR}=\mathrm{CR}+\mathrm{A} / \mathrm{CR}-\mathrm{Al}
$$


Os subíndices $x$, s e t representam os valores obtidos para o material em estudo, controle sensivei (IAC 899) e controle tolerante (IAC 25) respectivamente.

Milho

$$
\operatorname{ITR}=\left[\left(I C R_{x}-I C R_{s}\right) /\left(I C R_{t}-I C R_{s}\right)\right] \times 4+1
$$

onde:

ICR é o f́ndice de crescimento da radícula e representa o produto entre o comprimento relativo da radícula (CRR) e o comprimento relativo da raiz secundária mais longa (CRRSML). A concentração de Al foi de 4,5mg/litro. Assim sendo,

$$
\begin{gathered}
\text { ICR }=\text { CRR } \times \text { CRRSML, } \\
\text { CRR }=C R+A / C R-A, \\
\text { CRRSML }=\text { CRSML }+A / C R S M L-A
\end{gathered}
$$

Os subíndices $\mathrm{x}$, s e $\mathrm{t}$ representam os valores obtidos para o material em estudo, controle sensivel (HS7777) e controle tolerante (HS1227) respectivamente.

\section{RESULTADOS E DISCUSSÃO}

Com base no intervalo de confiança para as médias de IE e ITR, calculado com $99,9 \%$ de probabilidade, numa distribuição "t": Sx.t $(0,001 ; n)$, os genótipos de arroz e milho foram classificados quanto à eficiência a fósforo ou à tolerância a alumínio em nove classes:

I. Eficientes a fósforo, tolerantes a alumínio (ET);

II. Eficientes a fósforo, intermediários a alumínio (EI);

III. Eficientes a fósforo, não tolerantes a alumínio (ENT);

IV. Intermediários a fósforo, tolerantes a alumínio (IT);

V. Intermediários a fósforo, intermediários a alumínio (II);

VI. Intermediários a fósforo, não tolerantes a alumínio (INT);

VII. Não eficientes a fósforo, tolerantes a alumínio (NET);

VIII. Não eficientes a fósforo, intermediários a alumínio (NEI);

IX. Não eficientes a fósforo, não tolerantes a alumínio (NENT).

Os genótipos cujos valores de IE e ITR são superiores ao intervalo de confiança são eficientes $(E)$ e tolerantes $(T)$, enquanto aqueles que apresentam valores de IE e ITR inferiores ao intervalo de confiança são classificados em não eficientes (NE) e não tolerantes (NT). No caso de IE e ITR estarem no intervalo de confiança, os genótipos são classificados em intermediários (I) tanto à eficiência a fósforo quanto à tolerância a alumínio. 
Os resultados dos quadros 1 e 2 referem-se aos estudos com as linhagens de arroz de sequeiro e irrigado. Para cada tipo de arroz, os valores calculados para os índices de eficiência na absorção e uso do $P$ (IE), foram colocados em ordem crescente, ao lado dos índices de tolerância a Al, matéria seca das partes da planta e conteúdos de P. Outras variáveis estão relatadas em FURLANI et al. (1983), cujo trabalho mostra que as variações observadas nos parâmetros de eficiência a fósforo estiveram altamente correlacionadas com a produção de matéria seca, dentro de cada tipo de arroz, mas não estiveram correlacionadas com: número de folhas e de perfilhos por planta, ciclo e porte. Os autores observaram que os genótipos de arroz de sequeiro mostraram maior eficiência de extração ou de absorção do $\mathrm{P}$ da solução nutritiva $(4,47$ a $6,71 \mathrm{mg} P /$ vaso) em comparação com os de irrigado $(3,11$ a 5,67mg P/vaso), enquanto estes últimos mostraram maior eficiência na utilização do $\mathrm{P}$ para produção de matéria seca (arroz de sequeiro, $\mathrm{RE}=691$ a $1.470 \mathrm{mg}$ $\mathrm{MS} / \mathrm{mg} \mathrm{P}$; arroz irrigado = 1.061 a $2.005 \mathrm{mg} \mathrm{MS} / \mathrm{mg} \mathrm{P}$ ).

Observou-se que o índice IE, proposto por SIDDIQI \& GLASS (1981), expressa muito bem a variação observada na matéria seca e no conteúdo total de $P$. Entretanto, essa variável mostrou baixa correlação com a característica de seleção para tolerância a Al (ITR).

Embora a maioria de arroz de sequeiro tenha apresentado elevado índice de tolerância a $\mathrm{Al}$ (média ITR-Al $=4,15$ ), apenas onze linhagens se destacaram como eficientes a P e tolerantes a Al (ET): 76-100; IAC47; 75-10; 79-155; 79-105; 78-267; 79-233; 79-109; IAC165; IAC25 e 79-123.

Os resultados obtidos com 50 linhagens de arroz irrigado mostraram maior variabilidade na eficiência de utilização de $P$, evidenciada pela maior amplitude de variação entre as linhagens, para as variáveis determinadas (FURLANI et al., 1983) e para o índice IE calculado (Quadro 2). Também com relação à tolerância a Al, as linhagens de arroz irrigado mostraram maior variabilidade, indicando uma base genética de origem mais ampla para esse tipo de arroz. Entretanto, a correlação entre os dados de eficiência a $\mathrm{P}$ e os de tolerância a Al foi muito baixa.

Neste caso, a definição dos quadrantes de classificação das linhagens foi bem diversa daquela usada para o arroz de sequeiro. Embora muitas linhagens de arroz irrigado tenham apresentado altos valores absolutos de índices de eficiência a $\mathrm{P}$ (IE) (média IE $=11,01$ ), bem mais elevados do que os de arroz de sequeiro, a média para o índice de tolerância a Al (ITR-Al $=2,07$ ) foi bem inferior, e apenas duas das cinqüenta linhagens se destacaram como eficientes a $P$ e tolerantes a Al (ET): GI-74-27 e GI-74-29.

As linhagens de arroz de sequeiro apresentaram valores de IE menores que das de arroz irrigado (teste t 0,05 ), e estas, menores valores de ITR (teste t 0,05 ).

No quadro 3 estão os resultados com 40 linhagens de milho. $O$ índice de correlação de Spearman entre variáveis não paramétricas (STEEL \& TORRIE, 1960) (índices de eficiência de utilização de $\mathrm{P}$ e tolerância a $\mathrm{Al}$ ) foi muito baixo, negativo e não significativo, indicando uma independência entre os índices, embora a alguns 
QUADRO 1. Massa seca total, conteúdo total de $P$, indices de eficiência a $P$ (IE-P) e de tolerância relativa a Al (ITR-Al) de 50 genótipos de arroz de sequeiro

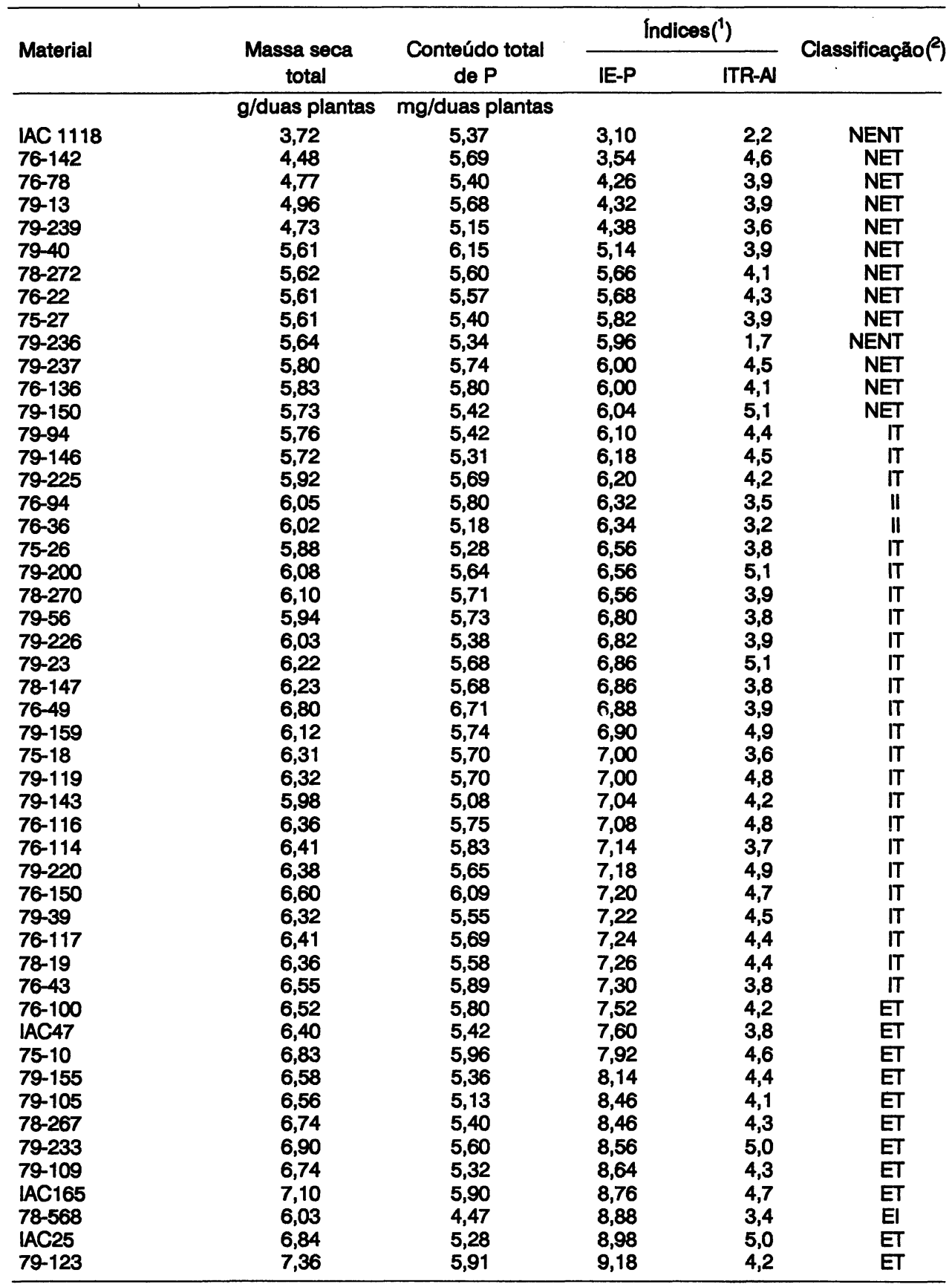

(') Intervalo de confiança para a média, calculada com $99,9 \%$ de probabilidade, numa distribuição "t": šx.t 0,001;49 para IE-P = 6,06 a 7,42 e "t": s̄.t o,001;99 para ITR-A = 2,66 a 3,56.

(2) Conforme descrito em resultados e discussão. 
QUADRO 2. Massa seca total, conteúdo total de $P$, índices de eficiência a P (IE-P) e de tolerância relativa a AI (ITR-Al) em 50 genótipos de arroz irrigado

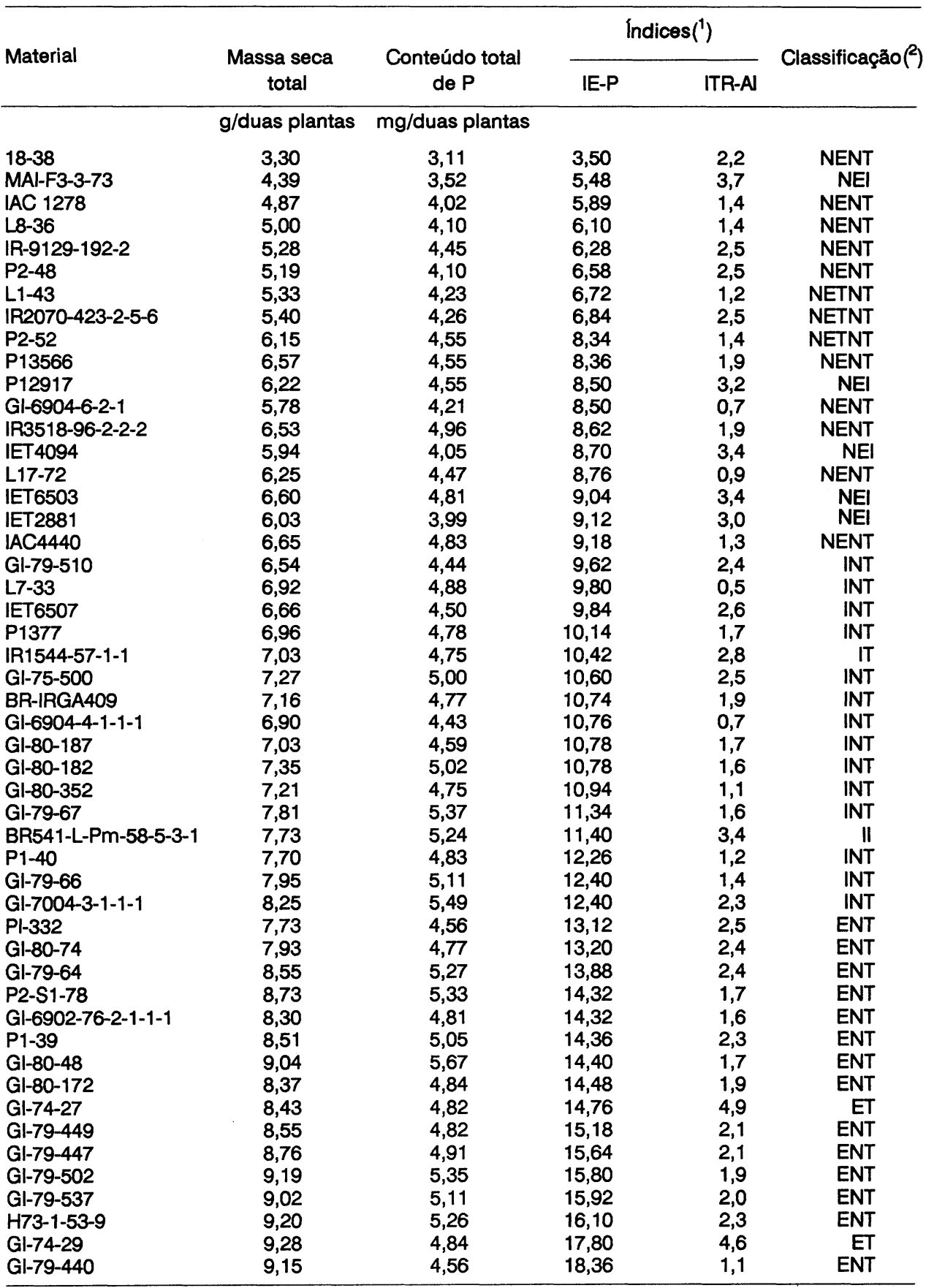

(1) Intervalo de confiança para a média, calculada com $99,9 \%$ de probabilidade, numa distribuiçăo

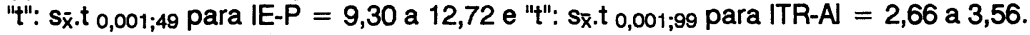

$\left({ }^{2}\right)$ Conforme descrito em resultados e discussão. 
QUADRO 3. Massa seca total, conteúdo total de $P$, índices de eficiência a P (IEP) e de tolerância relativa a Al (ITR-Al) de 40 linhagens de milho

\begin{tabular}{ccccc}
\hline Material & $\begin{array}{c}\text { Massa seca } \\
\text { total }\end{array}$ & $\begin{array}{c}\text { Conteúdo total } \\
\text { de } P\end{array}$ & Índices( $\left(^{1}\right)$ & \\
& Classificação( ${ }^{2}$ )
\end{tabular}

$\mathrm{g} /$ duas plantas $\mathrm{mg} / \mathrm{duas}$ plantas

\begin{tabular}{|c|c|c|c|c|c|}
\hline 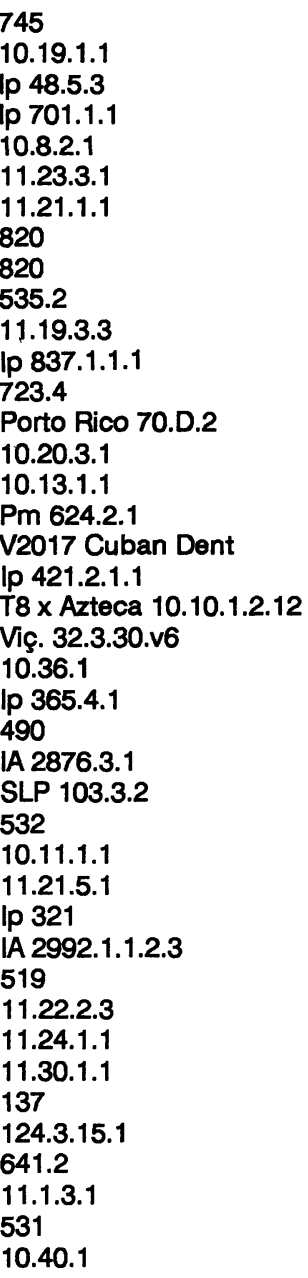 & $\begin{array}{l}4,46 \\
4,62 \\
4,64 \\
4,81 \\
5,12 \\
5,20 \\
5,81 \\
4,79 \\
4,79 \\
5,29 \\
5,30 \\
5,17 \\
5,40 \\
5,13 \\
5,51 \\
4,97 \\
5,25 \\
5,56 \\
5,34 \\
5,63 \\
5,65 \\
5,57 \\
5,51 \\
5,36 \\
5,73 \\
5,75 \\
5,49 \\
5,98 \\
5,83 \\
6,09 \\
5,79 \\
5,84 \\
6,32 \\
6,20 \\
6,92 \\
6,03 \\
6,41 \\
6,71 \\
6,84 \\
7,00 \\
7,40\end{array}$ & $\begin{array}{l}4,56 \\
4,89 \\
4,91 \\
4,80 \\
5,30 \\
5,30 \\
6,51 \\
4,41 \\
4,41 \\
5,34 \\
5,33 \\
4,93 \\
5,35 \\
4,78 \\
5,48 \\
4,42 \\
4,91 \\
5,50 \\
5,05 \\
5,24 \\
5,61 \\
5,44 \\
5,30 \\
4,98 \\
5,62 \\
5,59 \\
5,09 \\
5,91 \\
5,58 \\
6,08 \\
5,44 \\
5,42 \\
6,26 \\
5,80 \\
6,98 \\
5,20 \\
5,75 \\
5,98 \\
6,04 \\
5,78 \\
6,02\end{array}$ & $\begin{array}{l}4,36 \\
4,36 \\
4,38 \\
4,82 \\
4,95 \\
5,10 \\
5,18 \\
5,20 \\
5,20 \\
5,24 \\
5,27 \\
5,42 \\
5,45 \\
5,51 \\
5,54 \\
5,59 \\
5,61 \\
5,62 \\
5,65 \\
5,69 \\
5,69 \\
5,70 \\
5,73 \\
5,77 \\
5,84 \\
5,91 \\
5,92 \\
6,05 \\
6,09 \\
6,10 \\
6,16 \\
6,29 \\
6,38 \\
6,63 \\
6,86 \\
6,99 \\
7,15 \\
7,53 \\
7,75 \\
8,47 \\
9,10\end{array}$ & $\begin{array}{r}3,2 \\
2,0 \\
6,1 \\
0,7 \\
1,9 \\
2,8 \\
2,0 \\
5,1 \\
5,1 \\
11,0 \\
1,7 \\
0,5 \\
0,1 \\
5,2 \\
1,2 \\
1,1 \\
3,6 \\
0,5 \\
0,5 \\
0,5 \\
10,5 \\
1,3 \\
5,2 \\
7,0 \\
2,8 \\
0,5 \\
10,3 \\
1,3 \\
0,1 \\
2,6 \\
6,8 \\
4,9 \\
1,2 \\
1,5 \\
0,7 \\
0,1 \\
2,6 \\
3,0 \\
2,0 \\
0,9 \\
1,4\end{array}$ & $\begin{array}{r}\text { NEI } \\
\text { NEI } \\
\text { NET } \\
\text { NENT } \\
\text { NEI } \\
\text { NEI } \\
\text { NEI } \\
\text { NET } \\
\text { NET } \\
\text { NET } \\
\text { NEI } \\
\text { INT } \\
\text { INT } \\
\text { IT } \\
\text { INT } \\
\text { INT } \\
\text { II } \\
\text { INT } \\
\text { INT } \\
\text { INT } \\
\text { IT } \\
\text { INT } \\
\text { IT } \\
\text { IT } \\
\text { II } \\
\text { INT } \\
\text { IT } \\
\text { INT } \\
\text { INT } \\
\text { II } \\
\text { IT } \\
\text { IT } \\
\text { INT } \\
\text { EI } \\
\text { ENT } \\
\text { ENT } \\
\text { EI } \\
\text { EI } \\
\text { EI } \\
\text { ENT } \\
\text { ENT }\end{array}$ \\
\hline
\end{tabular}

(1) Intervalo de confiança para a média, calculada com $99,9 \%$ de probabilidade, numa distribuição

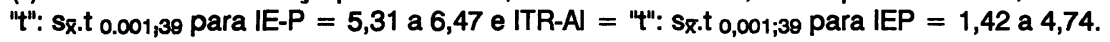

(2) Conforme descrito em resultados e discussão. 
valores altos tenham correspondido valores baixos de ITR, ou vice-versa. As linhagens interessantes seriam aquelas classificadas como intermediárias a $\mathrm{P}$ e tolerantes a Al (IT), neste caso em número de sete: Porto Rico 70.D.2; Viç. 32.2.30v.6; Ip 365.4.1; 490; 532; IA 2992.1.1.2.3 e 519.

Como fontes de tolerância a Al com elevados índices de ITR-Al, destacaram-se as linhagens: 532 (ITR-Al = 10,3); Viç. 32.3.30.V.6 (ITR-Al = 10,5) e 535.2 (NET; ITR-Al $=11,0$ ) (Quadro 3).

Na literatura são relatados os vários tipos de mecanismos envolvidos na tolerância a Al, e somente para algumas espécies parece ocorrer interação do Al com $\mathrm{P}, \mathrm{Ca}, \mathrm{K}$ e $\mathrm{Mg}$ que explique diferenças entre cultivares quanto a essa característica. FOY et al. (1967, 1973, 1978), em estudos com trigo e cevada, concluíram que a interação Al-P somente eventualmente explica diferenças entre plantas quanto à tolerância a Al. Em outros estudos com arroz, cevada, trigo e triticale, ficou evidente que plantas tolerantes a $\mathrm{Al}$ apresentavam teores mais altos de P, Ca, Mg e K (HOWELER \& CADAVID, 1976; MUGWIRA, 1980). Entretanto, essa informação não significa que tais plantas sejam efetivamente mais eficientes na absorção e utilização de $\mathrm{P}$. Pode ocorrer que maiores teores de $\mathrm{P}$ e de outros nutrientes encontrados nos tecidos sejam devidos ao maior aprofundamento $e$ crescimento do sistema radicular tolerante a $\mathrm{Al}$, que explora assim maior volume de solo.

O estudo de grande número de genótipos em função de duas variáveis (Al e P) fica dificultado. Entretanto, é imprescindfvel desenvolver mais pesquisa para o conhecimento da interação Al-P em cada espécie, para compreensão dos mecanismos fisiológicos envolvidos na tolerância a $\mathrm{Al}$ e eficiência a $\mathrm{P}$ e do controle genético aessas características.

É de grande importância a busca por plantas melhoradas com capacidade de adaptação a condições nutricionais adversas, na tentativa de amenizar os problemas encontrados nos solos brasileiros.

\section{CONCLUSÕES}

1) As linhagens de arroz de sequeiro mostraram-se superiores às de irrigado quanto à tolerância a $\mathrm{Al}$, e estas últimas, superiores às de sequeiro quanto à eficiência a $P$.

2) Das cem linhagens de arroz estudadas, onze de arroz de sequeiro e duas de irrigado destacaram-se como eficientes a $\mathrm{P}$ e tolerantes a Al.

3) Entre as quarenta linhagens de milho estudadas, três sobressaíram como tolerantes a Al; entretanto, intermediárias, quanto à eficiência a P.

4) Os baixos coeficientes de correlação (r) obtidos, entre os índices de eficiência a $P$ e tolerância a $A l$, para todas as linhagens de arroz e milho, são uma indicação de que essas características são independentes no material estudado. 


\section{REFERÊNCIAS BIBLIOGRÁFICAS}

CLARKSON, D.T. Effect of aluminum on the uptake and metabolism of phosphorus by barley seedlings. Plant Physlology, Lancaster, 41 (1):165-172, 1966.

FOY, C.D.; CHANEY, R.L. \& WHITE, M.C. The physiology of metal toxicity in plants. Annual Review of Plant Physiology, Palo Alto, 29:511-566, 1978.

GERLOFF, G.C. \& GABELMAN, W.H. Differential effects of aluminum on the vegetative growth of tomato cultivars in acid soil and nutrient solution. Journal of the American Soclety for Horticultural Sclence, New York, 98:427-432, 1973.

FURLANI, A.M.C.; BATAGLIA, O.C.; FURLANI, P.R., AZZINI, L.E. \& CAMARGO, O.B.A. Avaliaçăo de genótipos de arroz quanto à eficiência na utilizaçăo de fósforo em soluçăo nutritiva e em solo. Rovista Brasileira de Clência do Solo, Campinas, 7(3):291-303, 1983.

—_ — \& LIMA, M. Eficiência de linhagens de milho na absorção e utilizaçăo de fósforo em solução nutritiva. Bragantia, Campinas, 44(1):129-147, 1985.

\& FURLANI, P.R. Composição e pH de soluçōes nutritivas para estudos fisiológicos e seleção de plantas em condiçöes nutricionais adversas. Campinas, Instituto Agronômico, 1988. 34p. (Boletim técnico, 121)

FURLANI, P.R. \& HANNA, L.G. Avaliaçăo da tolerância de plantas de arroz $\theta$ milho ao alumínio em solução nutritiva. Revista Brasileira de Clêncla do Solo, Campinas, 8(2):205-208, 1984.

; LIMA, M; MIRANDA, L.T. de; MIRANDA, L.E.C. de; SAWAZAKI, E. \& MAGNAVAGA, R. Avaliação de linhagens, materiais comerciais e duas populaçőes de milho para tolerância a alumínio. Pesquisa Agropecuária Brasileira, Brasília, 21 (6):655-660, 1986.

HOWELER, R.H. \& CADAVID, L.F. Screening of rice cultivars for tolerance to A-toxicity in nutrient solutions as compared with a field screening method. Agronomy Journal, Madison, 68(4):551$555,1976$.

JONES, L.H. Aluminium uptake and toxicity in plants. Plant and Soll, The Hague, 13(4):297-310, 1961.

MUGWIRA, L.M.; ELGAWHARY, S.M. \& PATEL, K.I. Differential tolerances of triticale, wheat, rye, and barley to aluminum in nutrient solution. Agronomy Journal, Madison, 68(5):782-787, 1976.

; PATEL, S.U. \& FLEMING, A.L. Aluminium effects on growth and $\mathrm{A}, \mathrm{Ca}, \mathrm{Mg}, \mathrm{K}$ and $\mathrm{P}$ levels in triticale, wheat, and rye. Plant and Soll, The Hague, 57:467-470, 1980.

RHUE, R.D. Differential aluminum tolerance in crop plants. In: MUSSELL, H. \& STAPLES, R.C., eds. Stress physiology in crop plants. New York, John Wiley \& Sons, 1979. p.61-80.

SIDDIQI, M.Y. \& GLASS, A.D.M. Utilization index: a modified approach to the estimation and comparison of nutrient utilization efficiency in plants. Journal of Plant Nutrition, New York, 4(3):289-302, 1981.

STEEL, R.G.D. \& TORRIE, J.H. Principles and procedures of statistics. New York, MacGraw Hill, 1960. 481p. 